\title{
Observation of Defect-Free Surface Modes in Optical Waveguide Arrays
}

\author{
Alexander Szameit, ${ }^{1}$ Ivan L. Garanovich, ${ }^{2}$ Matthias Heinrich, ${ }^{1}$ Andrey A. Sukhorukov, ${ }^{2}$ Felix Dreisow, ${ }^{1}$ Thomas Pertsch, ${ }^{1}$ \\ Stefan Nolte, ${ }^{1}$ Andreas Tünnermann, ${ }^{1}$ and Yuri S. Kivshar ${ }^{2}$ \\ ${ }^{1}$ Institute of Applied Physics, Friedrich-Schiller-University Jena, Max-Wien-Platz 1, 07743 Jena, Germany \\ ${ }^{2}$ Nonlinear Physics Centre and Centre for Ultrahigh bandwidth Devices for Optical Systems (CUDOS), \\ Research School of Physical Sciences and Engineering, Australian National University, Canberra, ACT 0200, Australia
}

(Received 26 August 2008; revised manuscript received 3 October 2008; published 13 November 2008)

We report on the experimental observation of novel defect-free surface modes predicted theoretically for modulated photonic lattices [I. L. Garanovich et al., Phys. Rev. Lett. 100, 203904 (2008)]. We generate the linear surface modes in truncated arrays of periodically curved optical waveguides created in fused silica by a laser direct-writing technique. Our results demonstrate that the degree of surface wave localization can be controlled by selecting the waveguide bending amplitude.

PACS numbers: 42.25.Gy, 42.82.Et

Interfaces separating different physical media can support a special type of localized modes known as surface waves [1]. In solids, electronic surface waves generated in crystals at the edge of a truncated periodic potential are commonly explained as the manifestation of either Tamm [2] or Shockley [3] localization mechanisms. In optics, it was found that electromagnetic surface modes, which are similar to the electronic Tamm states, can exist at the interface separating periodic and homogeneous linear dielectric optical media [4], in contrast to the interfaces between homogeneous dielectrics, where a prohibitively high nonlinearity is required for the existence of surface waves [5,6]. Optical analogs of Tamm and Shockley surface states have also been studied extensively in different types of photonic crystal structures [7-14].

In discrete systems, such as arrays of weakly coupled optical waveguides [15], different types of linear and nonlinear states localized at and near the surface have also been analyzed. Whereas linear Shockley states can appear at the edges of superlattices without defects, their existence is limited to specific gaps $[3,14,16]$. In contrast, a surface defect is required for surface waves to exist in the photonic lattices composed of identical straight waveguides. It was found that Tamm surface waves can exist at the edge of an array of optical waveguides when the effective refractive index of the boundary waveguide is modified above a certain threshold [17-23].

In this Letter we report on the first experimental observation of novel defect-free surface modes predicted theoretically for modulated photonic lattices [24]. The modulated lattice is composed of weakly coupled optical waveguides which axes are periodically curved along the propagation direction $z$ [see schematic in Fig. 1(a)], and it can support well localized linear surface modes [24]. Such defect-free surface modes differ substantially from the commonly studied Tamm and Shockley surface states, as they appear despite the fact that all waveguides are identical, and there are no defects.
In order to study such defect-free surface modes experimentally, we use the femtosecond laser direct-writing technique to create periodically curved waveguide arrays in fused silica samples [25]. When ultrashort laser pulses are tightly focused into a transparent bulk material, nonlinear absorption takes place leading to optical breakdown and the formation of microplasma, which induces a permanent change in the material molecular structure. In the case of fused silica, the density is locally increased. Hence, by moving the sample transversely with respect to the beam, a continuous modification is obtained allowing to guide light [Fig. 1(b)]. This technique can be used to create large waveguiding structures with almost arbitrary topology $[26,27]$.

In our experiments, we fabricate curved waveguides with a sinusoidal bending profile of the form $x_{0}(z)=$ $A\{\cos [2 \pi z / L]-1\}$, where $x_{0}(z)$ is the transverse lattice shift as a function of the propagation distance $z, A$ and $L$ are the waveguide axes bending amplitude and period, respectively [see Fig. 1(a)]. When the bending amplitude is $A=A_{0}=\xi \lambda L / 4 \pi^{2} n_{0} d$ [marked point in Fig. 2(b)],

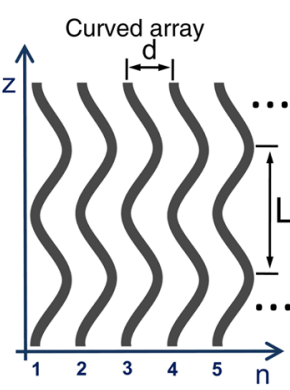

(a)

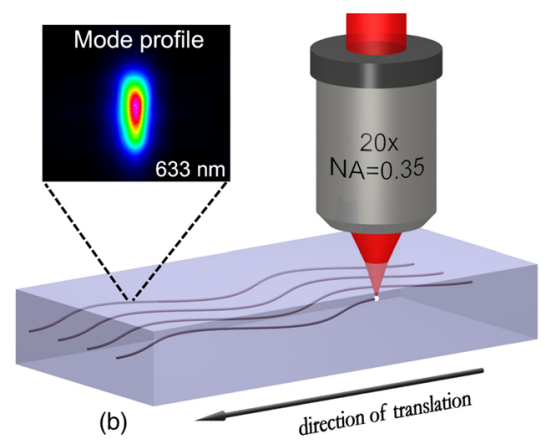

(b)
FIG. 1 (color online). (a) Periodically curved waveguide array with spacing $d$ and longitudinal bending period $L$. (b) Schematic of the femtosecond laser direct-writing setup. Insert shows waveguide mode profile measured at $633 \mathrm{~nm}$. 
(a)
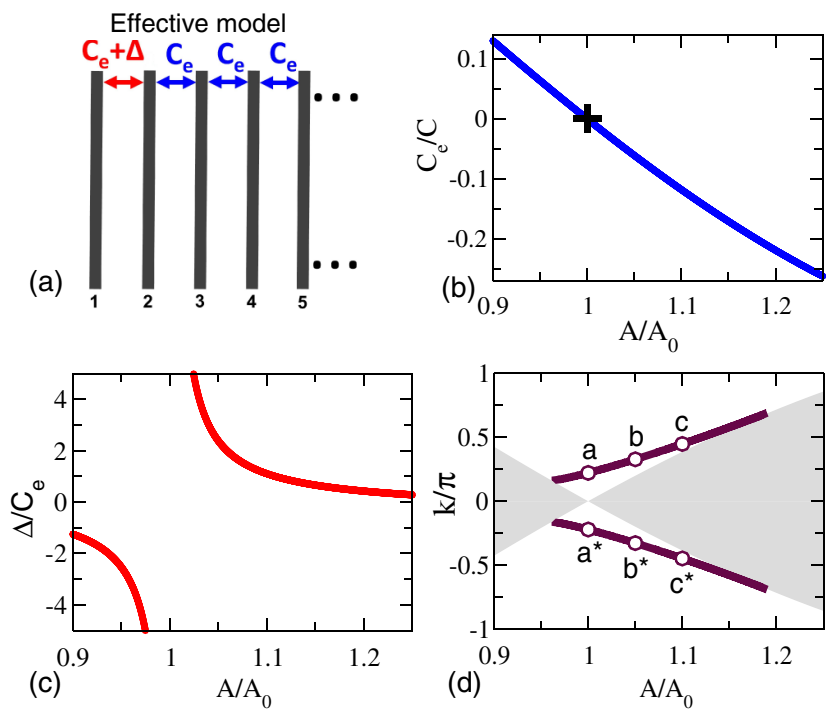

FIG. 2 (color online). (a) Effective model corresponding to the semi-infinite periodically curved waveguide array. (b) Effective coupling in infinite periodically curved array as a function of the bending amplitude. The self-collimation point is marked with a cross. (c) Effective surface defect strength in a semi-infinite periodically curved array as a function of the bending amplitude. (d) Numerically calculated propagation constants of the defectfree surface modes. Pairs of points marked by $a$ and $a^{*}, b$ and $b^{*}$, $c$ and $c^{*}$ correspond to Figs. 4(a)-4(c), respectively. The gray shading marks the lattice transmission band.

with $\lambda=633 \mathrm{~nm}$ as the vacuum wavelength, $n_{0}=1.45$ as the refractive index of the bulk silica glass, $d=14 \mu \mathrm{m}$ as the center-to- center spacing between adjacent waveguides, and $\xi \simeq 2.40$ as the first root of the Bessel function $J_{0}(\xi)[24,28]$, a beam launched in the center of the array experiences periodic self-imaging [28-31]. This is in close analogy to the dynamic localization of charged particles in ac electric fields [32].

For other bending amplitudes, light propagation in infinite [i.e., when the light beam is launched far away from the boundaries] sinusoidal waveguide arrays can be described by the effective coupling $C_{e}=C J_{0}\left(\xi A / A_{0}\right)$, where $C$ is the coupling coefficient in the straight waveguide array with the same waveguide spacing [24,28]. Such modification of the diffraction due to the periodic bending of waveguide axes is similar to the diffraction management which has been previously studied in different types of infinite modulated photonic lattices [28-31].

However, as has been recently predicted [24], in finitesize modulated lattices a periodic waveguide bending results in the nontrivial modification of the diffraction properties in the vicinity of the lattice edges, which is fundamentally different from the case of infinite modulated lattices. Specifically, light propagation in a semi-infinite periodically curved waveguide array can be described by the effective coupled equations [24], $i \frac{d u_{n}}{d z}+\left(C_{0}+\delta_{1, n} \Delta\right) u_{n+1}+\left(\bar{C}_{0}+\delta_{2, n} \bar{\Delta}\right) u_{n-1}=0$,

where $u_{n}(z)$ is the field amplitude in the $n$th waveguide, $n=1, \ldots, \infty$, and $u_{n \leq 0} \equiv 0$ due to the structure termination [i.e., the waveguide with $n=1$ corresponds to the surface of the semi-infinite lattice]. Here $\delta$ is the Kronecker delta, and the bar stands for the complex conjugation. Expression (1) shows that the periodic lattice modulation results in the appearance of the effective surface defect $\Delta$, which modifies the effective coupling strength between the edge waveguide and the rest of the lattice [see the schematic of the corresponding effective model in Fig. 2(a)]. We emphasize, that the actual structure does not have any real defects, as all the waveguides are identical with the same separation $d$. In the case of the sinusoidal lattice, the strength of the virtual surface defect can be calculated analytically by $\Delta=-\left(1 / 2 \pi^{2}\right) \times$ $C^{3} L^{2} J_{1}^{2}\left(\xi A / A_{0}\right) J_{2}\left(\xi A / A_{0}\right)$, where $J_{m}$ is the Bessel function of the first kind of the order $m$ [see Fig. 2(c)]. This quantity decreases when moving away from the selfcollimation point $A=A_{0}$ [Fig. 2(c)], and as a result the dynamical surface localization is possible in some finite interval of the bending amplitudes, even when diffraction is nonvanishing. This is in sharp contrast to the resonant self-collimation in infinite arrays, which takes place for only one particular value of the bending amplitude [see Fig. 2(b)]. Our samples are $70 \mathrm{~mm}$ long and contain 21 waveguides each. We choose the bending period to be $L=$ $23.33 \mathrm{~mm}$, so that each curved sample contains three full bending periods. Then the bending amplitude required for the self-collimation is $A_{0}=44.32 \mu \mathrm{m}$. In Fig. 2(d), the numerically calculated propagation constants $k$ of the surface modes are shown, which correspond to the measured coupling strength between the waveguides in our samples, $C=0.22 \mathrm{~mm}^{-1}$. The gray shading marks the lattice transmission band $|k| \leq 2\left|C_{e}\right| L$ [29], where localization is impossible.

In order to confirm the absence of surface defects in our samples, we first characterize the straight array. To directly observe the light propagation inside our samples we use a special fluorescence technique [33]. For the fabrication of the waveguides, fused silica with a high content of $\mathrm{OH}$ was used. This leads to a massive formation of nonbridging oxygen hole centers (NBOHCs) during the writing process, resulting in a homogeneous spatial distribution of these color centers along the waveguides. When launching light from a HeNe laser at $\lambda=633 \mathrm{~nm}$ into the waveguides, the NBOHCs are excited and the resulting fluorescence $(\lambda=$ $650 \mathrm{~nm}$ ) can be directly observed [34]. Since the color centers are formed exclusively inside the waveguides, this technique yields a high signal-to-noise ratio as the bulk material causes almost no background noise. In Figs. 3(a)3 (c) the fluorescence images of the light evolution in a straight array are shown when the beam is launched into the left edge waveguide $(n=1)$, the central waveguide 


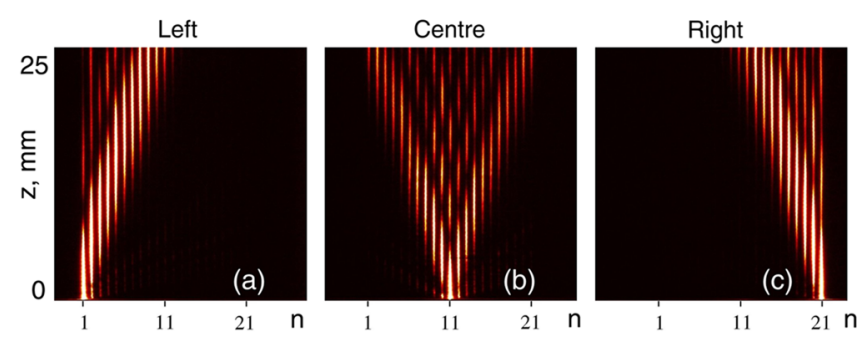

FIG. 3 (color online). Experimental fluorescent images of light propagation in the straight array. Laser beam is launched into the (a) left edge waveguide of the array, (b) center waveguide, and (c) right edge waveguide.

$(n=11)$, and the right edge waveguide $(n=21)$, respectively. When launched into the edge waveguides, the beam experiences strong repulsion from the surface and rapidly diffracts [Figs. 3(a) and 3(c)].

In order to study the generation of the defect-free waves, we have fabricated four curved waveguide arrays with bending amplitudes $A / A_{0}=1.00, A / A_{0}=1.05, A / A_{0}=$ 1.10 , and $A / A_{0}=1.20$. First, we study the light propaga-
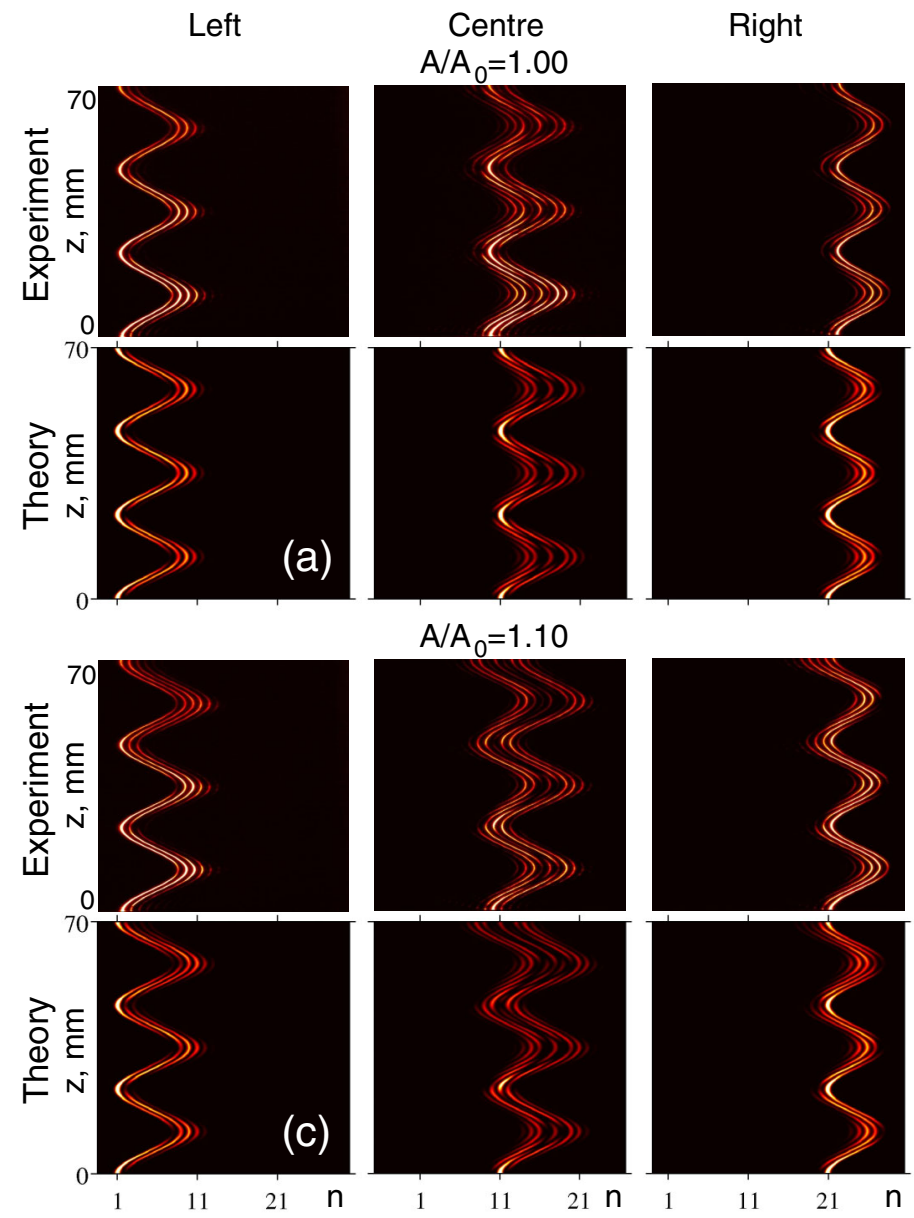

tion in the curved sample with $A / A_{0}=1.00$, which is tuned to the exact self-collimation. When the beam is launched into the left edge waveguide of the array, we observe the formation of a localized surface mode [Fig. 4(a), top left image] in excellent agreement with the corresponding numerical simulation [Fig. 4(a), bottom left image]. When we launch the beam into the array center, far away from the boundaries, we observe periodic beam selfcollimation [Fig. 4(a), center], similar to the previous experiments [28].

It has been predicted [24], that for symmetric waveguide bending profiles there exists an exact symmetry of the Eq. (1) such that for each solution $u_{n}(z)$ function $\tilde{u}_{n}(z)=$ $(-1)^{n} \bar{u}_{n}(z+L / 2)$ is also a solution. Therefore, in symmetric structures surface modes should always appear in pairs with the Bloch wave numbers of the opposite sign [see Fig. 2(d)]. When we launch the laser beam into the left edge waveguide of the array, the one of the two surface modes is generated which has a higher excitation coefficient at the input $z=0$ [i.e., the generated mode has most of its power concentrated in the first waveguide $(n=1)$ at the input], which is shown in Fig. 4(a), left. Because of the

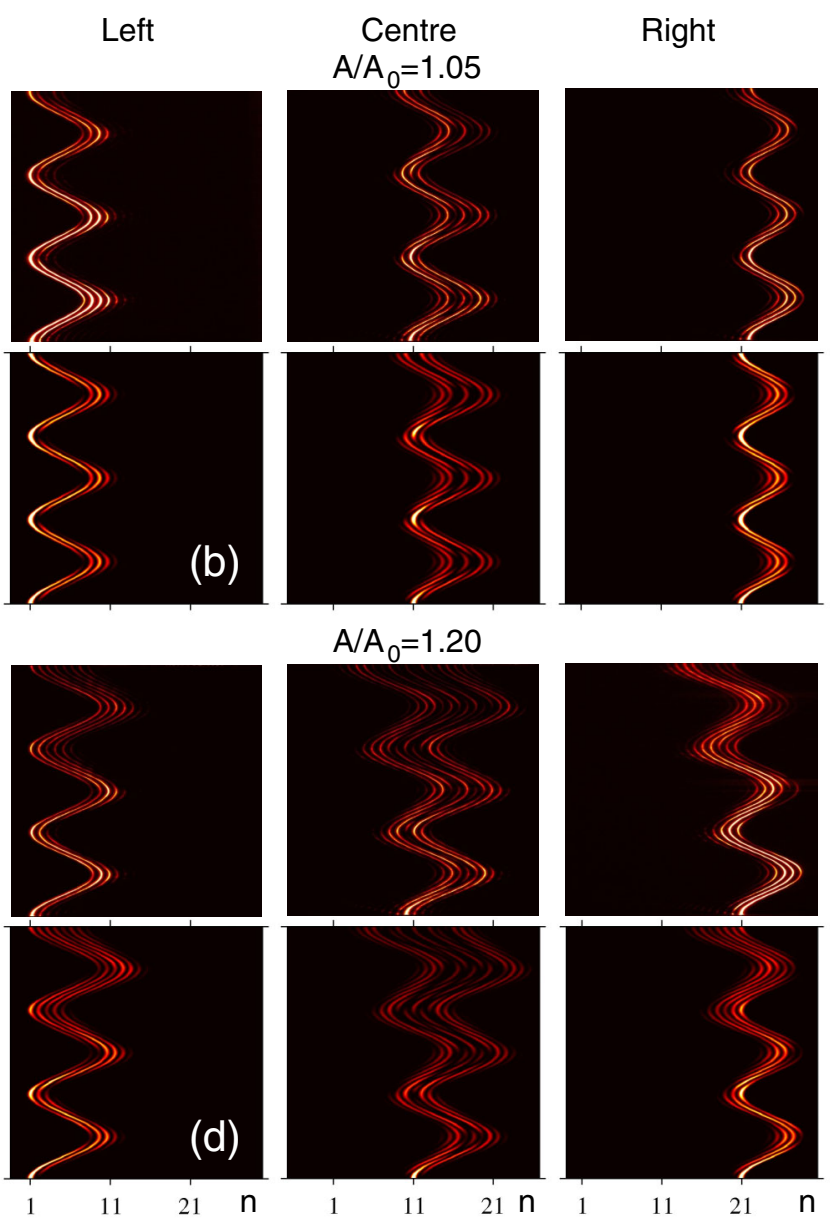

FIG. 4 (color online). (a-d) Light propagation in the four curved arrays with different bending amplitude $A$. In each of the four blocks fluorescent images are shown on the top, and corresponding numerical simulations are shown at the bottom. Light is launched into the left edge waveguide of the array (left), center waveguide (center), and right edge waveguide (right). 
symmetry of the Eq. (1), the second mode would have most of its power concentrated in the first waveguide at the distance $z=L / 2$. However, exciting the left edge waveguide at the distance $z=L / 2$ is equivalent to exciting the right edge waveguide $(n=21)$ at the distance $z=0$ because of the symmetry of our structure [see the sketch in Fig. 1(a)]. We excite the second mode by launching the laser beam into the right edge waveguide, as shown in Fig. 4(a), right. The two surface modes shown in Fig. 4(a), left, and Fig. 4(a), right, correspond to the points $a$ and $a^{*}$ in Fig. 2(d).

Next, we study the generation of surface waves in a curved array which bending amplitude is slightly detuned from the exact self-collimation value, $A / A_{0}=1.05$. When we launch the beam into the left and right array edges, we observe again the generation of two complementary surface modes [see Fig. 4(b), left; Fig. 4(b), right], which correspond to the points $b$ and $b^{*}$ in Fig. 2(d). In this case the generated surface waves are slightly less localized compared to the surface waves in Fig. 4(a), left, and Fig. 4(a), right, due to the increased initial radiation caused by the mismatch between the mode input profile and the single-site excitation which we use at the input. In contrast, when we launch the beam into the array center, we see significant beam diffraction [Fig. 4(b), center].

For the third sample with the bending amplitude $A / A_{0}=1.10$, excitation of the edge waveguides still yields the formation of defect-free surface waves [see Fig. 4(c), left; Fig. 4(c), right]. In this case, we observe much of the initial radiation, as the generated surface modes are quite close to the cutoff [points $c$ and $c^{*}$ in Fig. 2(d)]. When the array center is excited, strong diffraction is observed [Fig. 4(c), center].

The bending amplitude $A / A_{0}=1.20$ of the fourth sample is beyond the surface modes cutoff [see Fig. 2(d)], and we observe strong beam diffraction whether the beam is launched into the array center [Fig. 4(d), center] or into the edge waveguides [Figs. 4(d), left, and 4(d), right], in good agreement with the theoretical predictions.

In conclusion, we have demonstrated experimentally the effect of dynamical localization at the surface. We have generated defect-free linear surface modes at the edges of periodically curved optical waveguide arrays created in fused silica with the femtosecond laser direct-writing technique. We have employed fluorescent imaging to monitor directly the light propagation and demonstrated excellent agreement with the theoretical predictions. Our results reveal that the degree of surface wave localization can be effectively controlled by selecting the waveguide bending amplitude. The ability to generate these novel types of surface waves in optics can aid in the development of tools for the study of various surface phenomena. Similar effects can also occur in other types of modulated lattices in different physical systems.

The work was supported by the Australian Research Council and by the Leibnitz program of the Deutsche
Physikalische Gesellschaft.

[1] S. G. Davidson and M. Steslicka, Basic Theory of Surface States (Oxford Science Publications, New York, 1996).

[2] I. E. Tamm, Z. Phys. 76, 849 (1932).

[3] W. Shockley, Phys. Rev. 56, 317 (1939).

[4] P. Yeh, A. Yariv, and A. Y. Cho, Appl. Phys. Lett. 32, 104 (1978).

[5] W. J. Tomlinson, Opt. Lett. 5, 323 (1980).

[6] A. D. Boardman, P. Egan, F. Lederer, U. Langbein, and D. Mihalache, in Nonlinear Surface Electromagnetic Phenomena, Modern Problems in Condensed Matter Sciences, edited by H.E. Ponath and G. I. Stegeman (North-Holland, Amsterdam, 1991), Vol. 29, pp. 73-287.

[7] R.D. Meade, K.D. Brommer, and A. M. Rappe et al., Phys. Rev. B 44, 10961 (1991).

[8] F. Ramos-Mendieta and P. Halevi, Phys. Rev. B 59, 15112 (1999).

[9] J. M. Elson and K. Halterman, Opt. Express 12, 4855 (2004).

[10] Y. A. Vlasov, N. Moll, and S. J. McNab, Opt. Lett. 29, 2175 (2004).

[11] E. Moreno, F. J. García-Vidal, and L. Martín-Moreno, Phys. Rev. B 69, 121402 (2004).

[12] A. I. Rahachou and I. V. Zozoulenko, J. Opt. Soc. Am. B 23, 1679 (2006).

[13] S. K. Morrison and Yu. S. Kivshar, Opt. Commun. 266, 323 (2006).

[14] N. Malkova and C.Z. Ning, Phys. Rev. B 76, 045305 (2007).

[15] D. N. Christodoulides, F. Lederer, and Y. Silberberg, Nature (London) 424, 817 (2003).

[16] J. Zak, Phys. Rev. B 32, 2218 (1985).

[17] K. G. Makris et al., Opt. Lett. 30, 2466 (2005).

[18] M. I. Molina, Phys. Rev. B 71, 035404 (2005).

[19] S. Suntsov et al., Phys. Rev. Lett. 96, 063901 (2006).

[20] Y. V. Kartashov, V. A. Vysloukh, and L. Torner, Phys. Rev. Lett. 96, 073901 (2006).

[21] G. A. Siviloglou et al., Opt. Express 14, 5508 (2006).

[22] E. Smirnov et al., Opt. Lett. 31, 2338 (2006).

[23] C. R. Rosberg et al., Phys. Rev. Lett. 97, 083901 (2006).

[24] I. L. Garanovich, A. A. Sukhorukov, and Yu. S. Kivshar, Phys. Rev. Lett. 100, 203904 (2008).

[25] S. Nolte, M. Will, J. Burghoff, and A. Tuennermann, Appl. Phys. A 77, 109 (2003).

[26] A. Szameit et al., Opt. Lett. 33, 1542 (2008).

[27] A. Szameit et al., Phys. Rev. Lett. 98, 173903 (2007).

[28] S. Longhi et al., Phys. Rev. Lett. 96, 243901 (2006).

[29] H. S. Eisenberg, Y. Silberberg, R. Morandotti, and J. S. Aitchison, Phys. Rev. Lett. 85, 1863 (2000).

[30] I. L. Garanovich, A. A. Sukhorukov, and Yu. S. Kivshar, Phys. Rev. E 74, 066609 (2006).

[31] R. Iyer et al., Opt. Express 15, 3212 (2007).

[32] D. H. Dunlap and V. M. Kenkre, Phys. Rev. B 34, 3625 (1986).

[33] A. Szameit et al., Appl. Phys. Lett. 90, 241113 (2007).

[34] F. Dreisow et al., Opt. Express 16, 3474 (2008). 